\title{
Mean Field and Collisional Dynamics of Interacting Fermion-Boson Systems in a Soluble Model
}

\author{
E.R. Takano Natti* and A.F.R de Toledo Piza \\ Instituto de Física, Universidade de São Paulo, \\ C.P. 66318, 05389-970 São Paulo, SP, Brasil
}

July 26, 2018

\begin{abstract}
A general time-dependent projection technique is applied to the study of the dynamics of quantum correlations in a system consisting of interacting fermionic and bosonic subsystems, described by the Jaynes-Cummings Hamiltonian. The amplitude modulation of the Rabi oscillations which occur for a strong, coherent initial bosonic field is obtained from the spin intrinsic depolarization resulting from collisional corrections to the mean-field approximation.
\end{abstract}

PACS number(s): 03.70+k, 42.50.Md, 32.80-t

Keywords: Double mean-field approximation; Collisional dynamics; Time-dependent projection; Fermion-boson system.

\section{Introduction}

Even very simple open subsystems of closed quantum mechanical systems can display very intricate dynamical behavior, described by an effective, nonunitary time evolution law. The quantum state of each such subsystem can in

*E-MAIL: erica@fma.if.usp.br 
fact be described in terms of a reduced density operator which will in general evolve non-unitarily on the account of correlations and decoherence effects involving different subsystems [1, 2, 3]. The non-unitary effects will manifest themselves specifically through the dynamical evolution of the eigenvalues of the subsystem reduced density matrices, so that individual subsystems evolve in general in a non-isoentropic fashion.

A first mean-field-like approximation to this general picture consists in assuming isoentropic subsystem evolution under effective, time-dependent Hamiltonian operators for each subsystem. This approximation can be made self-consistent by allowing for explicit dependence of each subsystem effective Hamiltonian on the present state of the other, external subsystem [1]. Even when such selfconsistency requirement is abandoned by simply parametrizing the externally induced time dependence of the relevant subsystem effective Hamiltonian, one is still left in general with a nonlinear dynamical problem which defies exact treatment in a many-body or quantum field theoretical context. In this case, the subsystem time evolution can be analysed under a further mean field approximation through a variational principle by making a Gaussian Ansatz for the subsystem density matrix [2].

In this work we implement the fully self-consistent double time-dependent mean field approximation to a simple model system consisting of interacting fermionic and bosonic subsystems described by the Jaynes-Cummings Hamiltonian [⿴囗十 . Furthermore, by using the projection approach developed by Nemes and Toledo Piza [1] we obtain and evaluate explicitly corrections to this self consistent mean field description. These corrections appear in the form of suitable memory integrals added to the mean-field dynamical equations. The resulting dynamical equations acquire then the structure of kinetic equations, with the memory integrals performing as collision terms which eliminate the isoentropic mean-field constraint. Relevance of the JaynesCummings model in this context stems also from the fact that, besides being soluble, it can be seen as corresponding to the relativistic scalar plasma [5] in zero spatial dimensions. The study of soluble models provides a clear understanding of the physical phenomena involved and is useful in controlling various approximations necessary for the treatment of more realistic cases.

The definition of the relevant variables and our approach for studying the dynamics of these variables are described in section 2 . In section 3 we obtain the dynamical equations for these variables in the collisional approximation . Finally we show and discuss some numerical results, comparing to the exact 
solution of the model.

\section{Gaussian Variables and Their Effective Dy- namics}

The Jaynes-Cummings Model (JCM) [4] describes the simplest fully quantized version of a system consisting of two interacting quantum subsystems of different nature: a two level atom (fermionic system) and a quantized field mode (bosonic system). The corresponding exactly soluble Hamiltonian is given by

$$
H=\frac{\epsilon}{2}\left(a_{1}^{\dagger} a_{1}-a_{-1}^{\dagger} a_{-1}\right)+\omega b^{\dagger} b+\lambda\left(a_{1}^{\dagger} a_{-1} b+a_{-1}^{\dagger} a_{1} b^{\dagger}\right),
$$

where the annihilation and creation operators $b$ and $b^{\dagger}$ satisfy the boson commutation relation $\left[b, b^{\dagger}\right]=1$ and the fermion operators $a_{\lambda}$ and $a_{\lambda}^{\dagger}$ satisfy the anticommutation relations $\left\{a_{\lambda}, a_{\lambda^{\prime}}^{\dagger}\right\}=\delta_{\lambda^{\prime}, \lambda}$. The solubility of the model stems from the fact that the interaction term of $H$ is written in the so called Rotating Wave Approximation, which consists in ignoring "anti-ressonant" terms involving $a_{1}^{\dagger} a_{-1} b^{\dagger}$ and its Hermitian conjugate.

The general idea of our approach is to focus on the time evolution of variables which are fully determined by the mean values of the annihilation/creation operators and their bilinear or quadratic combinations, henceforth refered to as gaussian observables. The state of the system is generally given in terms of a density matrix $\mathcal{F}$ in the Heisenberg picture. $\mathcal{F}$ is hermitian, time independent and has unit trace.

The mean values of the boson operators are given by

$$
\langle b(t)\rangle \equiv \mathcal{B}_{t}=\operatorname{Tr}_{\mathrm{BF}} b(t) \mathcal{F}
$$

and its complex conjugate. Here and in what follows the symbol $T r_{\mathrm{BF}}$ denotes a trace over both bosonic and fermionic variables. Partial traces over bosonic or fermionic variables will be written as $T r_{\mathrm{B}}$ and $T r_{\mathrm{F}}$ respectively. Using them we can define the shifted boson operators

$$
d(t) \equiv b(t)-\mathcal{B}_{t}
$$

which have vanishing $\mathcal{F}$-expectation values. 
Next, the mean values of the bilinear forms of boson operators can be combined in an extended one boson plus pairing density matrix $\mathcal{R}[$ [

$$
\mathcal{R}_{\mathrm{B}}=\left[\begin{array}{cc}
R_{\mathrm{B}} & \Pi_{\mathrm{B}} \\
\Pi_{\mathrm{B}}^{*} & 1+R_{\mathrm{B}}
\end{array}\right]=\left[\begin{array}{cc}
\left\langle d^{\dagger}(t) d(t)\right\rangle & \langle d(t) d(t)\rangle \\
\left\langle d^{\dagger}(t) d^{\dagger}(t)\right\rangle & \left\langle d(t) d^{\dagger}(t)\right\rangle
\end{array}\right]
$$

where the one-boson density matrix $R_{\mathrm{B}}$ is hermitian and the pairing density matrix $\Pi_{\mathrm{B}}$ is symmetric.

For the fermionic system the extended density matrix is

$$
\mathcal{R}_{\mathrm{F}}=\left[\begin{array}{cc}
R_{\mathrm{F}} & \Pi_{\mathrm{F}} \\
-\Pi_{\mathrm{F}}^{*} & 1-R_{\mathrm{F}}
\end{array}\right]=\left[\begin{array}{cc}
\left\langle a_{\lambda^{\prime}}^{\dagger}(t) a_{\lambda}(t)\right\rangle & \left\langle a_{\lambda^{\prime}}(t) a_{\lambda}(t)\right\rangle \\
\left\langle a_{\lambda^{\prime}}^{\dagger}(t) a_{\lambda}^{\dagger}(t)\right\rangle & \left\langle a_{\lambda^{\prime}}(t) a_{\lambda}^{\dagger}(t)\right\rangle
\end{array}\right]
$$

where the hermitian matrix $R_{\mathrm{F}}$ and the antisymmetric matrix $\Pi_{\mathrm{B}}$ are the one-fermion and pairing density matrices, respectively.

To deal with the pairing densities one defines new Bogoliubov quasiparticle operators. In the case of the bosons we define quasi-boson operators as

$$
\begin{aligned}
\beta(t) & =x_{t}^{*}\left(b-\mathcal{B}_{t}\right)+y_{t}^{*}\left(b^{+}-\mathcal{B}_{t}^{*}\right) \\
\beta^{+}(t) & =x_{t}\left(b^{+}-\mathcal{B}_{t}^{*}\right)+y_{t}\left(b-\mathcal{B}_{t}\right)
\end{aligned}
$$

and require that $\langle\beta \beta\rangle=\left\langle\beta^{\dagger} \beta^{\dagger}\right\rangle=0$. The preservation of the commutation relations requires furthermore that the transformation coefficients $x_{t}$ and $y_{t}$ be chosen so that $\left|x_{t}\right|^{2}-\left|y_{t}\right|^{2}=1$. Thus the densities $\left\langle b^{\dagger} b^{\dagger}\right\rangle$ and $\langle b b\rangle$ can be parametrized in terms of $x_{t}, y_{t}$ and $\nu=\left\langle\beta^{\dagger} \beta\right\rangle$.

The coefficients $x_{t}$ and $y_{t}$ of the Bogoliubov transformation are determined by solving the secular problem

$$
G \mathcal{R}_{\mathrm{B}} X_{\mathrm{B}}=X_{\mathrm{B}} G Q_{\mathrm{B}}
$$

where 


$$
X_{\mathrm{B}}=\left[\begin{array}{cc}
x_{t} & y_{t}^{*} \\
y_{t} & x_{t}^{*}
\end{array}\right] \quad G=\left[\begin{array}{cc}
1 & 0 \\
0 & -1
\end{array}\right] \quad Q_{\mathrm{B}}=\left[\begin{array}{cc}
\nu & 0 \\
0 & 1+\nu
\end{array}\right] .
$$

The eigenvalue $\nu$ can be interpreted as shifted boson occupation number for the paired natural orbital described by $X_{\mathrm{B}}$. Since the Bogoliubov transformation is canonical one can verify that $X_{\mathrm{B}}$ satisfies the orthogonality and completeness relation

$$
X_{\mathrm{B}}^{\dagger} G X_{\mathrm{B}}=X_{\mathrm{B}} G X_{\mathrm{B}}^{\dagger}=G
$$

For the fermion operators we define the transformation

$$
\left[\begin{array}{c}
\alpha_{1}(t) \\
\alpha_{-1}(t) \\
\alpha_{1}^{\dagger}(t) \\
\alpha_{-1}^{\dagger}(t)
\end{array}\right]=\left[\begin{array}{cc}
X^{*} & 0 \\
0 & X
\end{array}\right]\left[\begin{array}{c}
a_{1}(t) \\
a_{-1}(t) \\
a_{1}^{\dagger}(t) \\
a_{-1}^{\dagger}(t)
\end{array}\right]
$$

where

$$
X=\left[\begin{array}{cc}
u_{t} & -v_{t} \\
v_{t}^{*} & u_{t}^{*}
\end{array}\right]
$$

Here we require that $\left\langle\alpha_{\lambda^{\prime}}(t) \alpha_{\lambda}(t)\right\rangle=\left\langle\alpha_{\lambda^{\prime}}^{\dagger}(t) \alpha_{\lambda}^{\dagger}(t)\right\rangle=0$ and the fermion densities $\left\langle a_{\lambda^{\prime}}^{\dagger}(t) a_{\lambda}(t)\right\rangle$ and $\left\langle a_{\lambda^{\prime}}(t) a_{\lambda}^{\dagger}(t)\right\rangle$ are parametrized in terms of $u_{t}, v_{t}$ and $p_{\lambda}=\left\langle\alpha_{\lambda}^{\dagger} \alpha_{\lambda}\right\rangle, \lambda=-1,1$. As in the case of the bosons we have an eigenvalue problem

$$
\mathcal{X}_{\mathrm{F}}^{\dagger} \mathcal{R}_{\mathrm{F}} \mathcal{X}_{\mathrm{F}}=Q_{\mathrm{F}}
$$

where $\mathcal{X}_{\mathrm{F}}$ is the transformation defined in (9)

$$
\mathcal{X}_{\mathrm{F}}=\left[\begin{array}{cc}
X^{*} & 0 \\
0 & X
\end{array}\right] \text { and } \mathcal{X}_{\mathrm{F}}^{\dagger}=\left[\begin{array}{cc}
X^{T} & 0 \\
0 & X^{\dagger}
\end{array}\right]
$$


satisfying the unitary condition

$$
\mathcal{X}_{\mathrm{F}}^{\dagger} \mathcal{X}_{\mathrm{F}}=\mathcal{X}_{\mathrm{F}} \mathcal{X}_{\mathrm{F}}^{\dagger}=\mathbf{I}_{4}
$$

The matrix $Q_{\mathrm{F}}$ is the extended density matrix in quasi-particle basis

$$
Q_{\mathrm{F}}=\left[\begin{array}{cc}
\mathbf{p}_{\lambda} & 0 \\
0 & 1-\mathbf{p}_{\lambda}
\end{array}\right]=\left[\begin{array}{cc}
\left\langle\alpha_{\lambda^{\prime}}^{\dagger}(t) \alpha_{\lambda}(t)\right\rangle & 0 \\
0 & \left\langle\alpha_{\lambda^{\prime}}(t) \alpha_{\lambda}^{\dagger}(t)\right\rangle
\end{array}\right]
$$

where $\mathbf{p}_{\lambda}$ is the one-fermion density matrix in quasi-particle basis. In this basis $\mathbf{p}_{\lambda}$ is diagonal with eigenvalues $p_{1}$ and $p_{-1}$ which correspond to the occupation numbers of the natural orbitals.

The next step is to obtain the time evolution of the mean values of gaussian observables. We begin with the boson subsystem. For $\mathcal{B}_{t}$ one finds immediately

$$
i \dot{\mathcal{B}}_{t}=\operatorname{Tr}_{\mathrm{BF}}[b, H] \mathcal{F}=x_{t} \operatorname{Tr}_{\mathrm{BF}}[\beta, H] \mathcal{F}-y_{t}^{*} \operatorname{Tr}_{\mathrm{BF}}\left[\beta^{\dagger}, H\right] \mathcal{F}
$$

where $H$ is the Hamiltonian given by (1). For the remaining quantities, we first rewrite the eigenvalue equation (6), using (8), as

$$
X_{\mathrm{B}}^{\dagger} \mathcal{R}_{\mathrm{B}} X_{\mathrm{B}}=Q_{\mathrm{B}}
$$

Taking the time derivative we have

$$
\begin{aligned}
X_{\mathrm{B}}^{\dagger} \dot{\mathcal{R}}_{\mathrm{B}} X_{\mathrm{B}} & =\dot{Q}_{\mathrm{B}}-\dot{X}_{\mathrm{B}}^{\dagger} \mathcal{R}_{\mathrm{B}} X_{\mathrm{B}}-X_{\mathrm{B}}^{\dagger} \mathcal{R}_{\mathrm{B}} \dot{X}_{\mathrm{B}} \\
& =\dot{Q}_{\mathrm{B}}-\dot{X}_{\mathrm{B}}^{\dagger} G X_{\mathrm{B}} G Q_{\mathrm{B}}-Q_{\mathrm{B}} G X_{\mathrm{B}}^{\dagger} G \dot{X}_{\mathrm{B}}
\end{aligned}
$$

The left hand side of this equation can be evaluated using the Heisenberg equation of motion

$$
i X_{\mathrm{B}}^{\dagger} \dot{\mathcal{R}}_{\mathrm{B}} X_{\mathrm{B}}=\left(\begin{array}{cc}
\operatorname{Tr}_{\mathrm{BF}}\left[\beta^{\dagger} \beta, H\right] \mathcal{F} & \operatorname{Tr}_{\mathrm{BF}}[\beta \beta, H] \mathcal{F} \\
\operatorname{Tr}_{\mathrm{BF}}\left[\beta^{\dagger} \beta^{\dagger}, H\right] \mathcal{F} & \operatorname{Tr}_{\mathrm{BF}}\left[\beta \beta^{\dagger}, H\right] \mathcal{F}
\end{array}\right)
$$


The right hand side of eq. (17), on the other hand can be rewritten using (7). Equating the result to (18) we have equations which describe the time evolution of the bosonic variables explicitly :

$$
\begin{aligned}
i \dot{\nu} & =\operatorname{Tr}_{\mathrm{BF}}\left[\beta^{\dagger} \beta, H\right] \mathcal{F} \\
i(1+2 \nu)\left(\dot{x}_{t}^{*} y_{t}^{*}-x_{t}^{*} \dot{y}_{t}^{*}\right) & =\operatorname{Tr}_{\mathrm{BF}}[\beta \beta, H] \mathcal{F} .
\end{aligned}
$$

We have next to obtain analogous equations for the fermionic variables. Taking the time derivative of the eigenvalue equation (11) and using the unitary condition (13) we obtain

$$
\mathcal{X}_{\mathrm{F}} \dot{\mathcal{R}}_{\mathrm{F}} \mathcal{X}_{\mathrm{F}}=\dot{Q}_{\mathrm{F}}-\dot{\mathcal{X}}_{\mathrm{F}}^{\dagger} \mathcal{X}_{\mathrm{F}} Q_{\mathrm{F}}-Q_{\mathrm{F}} \mathcal{X}_{\mathrm{F}}^{\dagger} \dot{\mathcal{X}}_{\mathrm{F}}
$$

Substituting (12) and (14) and using the unitary condition (13) we can evaluate the right hand side of (21). To evaluate the left hand side we use the Heisenberg equation of motion. We obtain

$$
\begin{aligned}
i \dot{p}_{\lambda} & =\operatorname{Tr}_{\mathrm{BF}}\left[\alpha_{\lambda}^{\dagger} \alpha_{\lambda}, H\right] \mathcal{F}, \quad \lambda=-1,1 \\
i\left(p_{1}-p_{-1}\right)\left(\dot{u}_{t} v_{t}-\dot{v}_{t} u_{t}\right) & =\operatorname{Tr}_{\mathrm{BF}}\left[\alpha_{1}^{\dagger} \alpha_{-1}, H\right] \mathcal{F}
\end{aligned}
$$

which describe the dynamics of the fermionic variables.

Eqs. (15), (19), (20), (22) and (23) are however not closed, $\mathcal{F}$ is not fully determined by gaussian variables. In order to deal with this situation we split the full density as

$$
\begin{aligned}
\mathcal{F} & =\mathcal{F}_{0}(t)+\mathcal{F}^{\prime}(t) \\
& \equiv \mathcal{F}_{0}^{\mathrm{B}} \mathcal{F}_{0}^{\mathrm{F}}+\mathcal{F}^{\prime}(t)
\end{aligned}
$$

where the factorized form of $\mathcal{F}_{0}(t)$ embodies what we refer to as the double mean field approximation. The subsystem densities $\mathcal{F}_{0}^{\mathrm{B}}$ and $\mathcal{F}_{0}^{\mathrm{F}}$ are in fact 
gaussian densities, written in the the form of an exponential of a bilinear, Hermitian expression in the creation/annihilation parts of the bosonic and of the fermionic fields respectively. When explicitly normalized to unit trace they are given in terms of the transformed operators introduced in Eqs. (5) and (9) as [7, 10]

$$
\begin{aligned}
\mathcal{F}_{0}^{\mathrm{F}} & =\prod_{\lambda}\left[p_{\lambda} \alpha_{\lambda}^{\dagger} \alpha_{\lambda}+\left(1-p_{\lambda}\right) \alpha_{\lambda} \alpha_{\lambda}^{\dagger}\right] \\
\mathcal{F}_{0}^{\mathrm{B}} & =\frac{1}{1+\nu}\left(\frac{\nu}{1+\nu}\right)^{\beta^{\dagger} \beta} .
\end{aligned}
$$

The remainder density $\mathcal{F}^{\prime}(t)$ is consequently a traceless, pure correlation part of the full density. In view of the special form used for $\mathcal{F}_{0}(t)$ it will in general contain correlations of two types : inter-subsystem (boson-fermion) correlations and intra-subsystem (boson-boson and fermion-fermion) correlations.

The next step consists in using the fact that the decomposition (24) can be implemented in terms of a time-dependent projection operator $\mathcal{P}(t)=$ $\mathcal{P}(t) \mathcal{P}(t)$ (cf. Ref. [8])

$$
\mathcal{P} \cdot=\mathcal{F}_{0}^{\mathrm{F}} \operatorname{Tr}_{\mathrm{F}} \mathcal{P}_{\mathrm{B}} \cdot+\mathcal{F}_{0}^{\mathrm{B}} \operatorname{Tr}_{\mathrm{B}} \mathcal{P}_{\mathrm{F}} \cdot-\mathcal{F}_{0}^{\mathrm{F}} \mathcal{F}_{0}^{\mathrm{B}} \operatorname{Tr}_{\mathrm{F}} \operatorname{Tr}_{\mathrm{B}}
$$

where $\mathcal{P}_{\mathrm{B}}$ is the boson projection operator 11

$$
\begin{aligned}
\mathcal{P}_{\mathrm{B}} \cdot & =\left[\left(1-\frac{\beta^{\dagger} \beta-\nu}{1+\nu}\right) \operatorname{Tr}_{\mathrm{B}} \cdot+\frac{\beta^{\dagger} \beta-\nu}{\nu(1+\nu)} \operatorname{Tr}_{\mathrm{B}}\left(\beta^{\dagger} \beta \cdot\right)+\frac{\beta}{\nu} \operatorname{Tr}_{\mathrm{B}}\left(\beta^{\dagger} \cdot\right)+\right. \\
& \left.+\frac{\beta^{\dagger}}{1+\nu} \operatorname{Tr}_{\mathrm{B}}(\beta \cdot)+\frac{\beta \beta}{2 \nu^{2}} \operatorname{Tr}_{\mathrm{B}}\left(\beta^{\dagger} \beta^{\dagger} \cdot\right)+\frac{\beta^{\dagger} \beta^{\dagger}}{2(1+\nu)^{2}} \operatorname{Tr}_{\mathrm{B}}(\beta \beta \cdot)\right] \mathcal{F}_{0}^{\mathrm{B}},
\end{aligned}
$$

$\mathcal{P}_{\mathrm{F}}$ is the fermion projector 9

$\mathcal{P}_{\mathrm{F}} \cdot=\left[\left(1-\sum_{\lambda=-1,1} \frac{\alpha_{\lambda}^{\dagger} \alpha_{\lambda}-p_{\lambda}}{1-p_{\lambda}}\right) \operatorname{Tr}_{\mathrm{F}} \cdot+\sum_{\lambda, \lambda=-1,1}\left(\frac{\alpha_{\lambda}^{\dagger} \alpha_{\lambda \prime}-p_{\lambda} \delta_{\lambda \lambda^{\prime}}}{p_{\lambda \prime}\left(1-p_{\lambda}\right)}\right) \operatorname{Tr}_{\mathrm{F}}\left(\alpha_{\lambda^{\prime}}^{\dagger} \alpha_{\lambda} \cdot\right)\right] \mathcal{F}_{0}^{\mathrm{F}}$

and the dot stands for the object on which the operator acts. With the help of $\mathcal{P}(t)$ the factorized gaussian density can be obtained as 


$$
\mathcal{F}_{0}(t)=\mathcal{P}(t) \mathcal{F}
$$

while its time-derivative is given by the expression

$$
i \dot{\mathcal{F}}_{0}(t)=\left[\mathcal{F}_{0}(t), H\right]+\mathcal{P}(t)[H, \mathcal{F}]
$$

which is in fact the Heisenberg Picture counterpart of the equation $\dot{\mathcal{P}} \mathcal{F}=0$ which has been used to define $\mathcal{P}(t)$ in Schrödinger Picture [8, 9].

The remaining formal steps towards closing the equations of motion for the gaussian variables are now straightforward. From (24) and (31) we can obtain a differential equation for $\mathcal{F}^{\prime}(t)$ in terms of $\mathcal{F}_{0}(t)$

$$
\left[i \frac{d}{d t}+\mathcal{P}(t) \mathcal{L}\right] \mathcal{F}^{\prime}(t)=\mathcal{Q} \mathcal{L F}_{0}(t)
$$

where we introduced the complementary projector $\mathcal{Q}(t) \mathcal{F} \equiv(\mathcal{I}-\mathcal{P}(t)) \mathcal{F}=$ $\mathcal{F}^{\prime}(t)$ and the notation $\mathcal{L}$. for the Liouvillian time-displacement generator $[H, \cdot]$. This equation has the formal solution

$$
\mathcal{F}^{\prime}(t)=\mathcal{G}(t, 0) \mathcal{F}^{\prime}(0)-i \int_{0}^{t} d t^{\prime} \mathcal{G}\left(t, t^{\prime}\right) \mathcal{Q}\left(t^{\prime}\right) \mathcal{L} \mathcal{F}_{0}\left(t^{\prime}\right)
$$

where the first term accounts for initial conditions, and $\mathcal{G}\left(t, t^{\prime}\right)$ is the timeordered Green's function

$$
\mathcal{G}\left(t, t^{\prime}\right)=\mathrm{T} \exp \left[i \int_{t^{\prime}}^{t} d \tau \mathcal{P}(\tau) \mathcal{L}\right]
$$

Thus $\mathcal{F}^{\prime}(t)$, and therefore $\mathcal{F}$, can be formally expressed in terms of $\mathcal{F}_{0}\left(t^{\prime}\right)$ for $t^{\prime}<t$ and of initial correlations $\mathcal{F}^{\prime}(0)$. This finally allows one to express the dynamical equations (15), (19), (20), (22), and (23) as traces over functionals of $\mathcal{F}_{0}\left(t^{\prime}\right)$ and of the initial correlation part $\mathcal{F}^{\prime}(0)$.

\section{Approximation to the Collision Dynamics}

As can be seen from the formally exact Eq. (33), the correlation contributions to the dynamical equations involve traces over functionals of $\mathcal{F}_{0}\left(t^{\prime}\right)$ with 
memory effects besides the initial correlations. Since these objects involve the complicated fully correlated time-dependence of the Heisenberg operators, approximations are clearly needed for their actual evaluation.

A systematic expansion scheme for the memory effects in which the mean energy is conserved in all orders has been discussed in ref. [9] in terms of the Schrödinger Picture. Following Lin [11] we implement in the Heisenberg picture a modified version of the lowest order approximation given in that work which consists in approximating the actual time evolution of the operators $\beta(t)$ when evaluating memory effects, by the effective mean field evolution

$$
i \frac{d \beta}{d t}=\left[\beta, H_{0}\right]+i\left(\dot{x}_{t}^{*} x_{t}-\dot{y}_{t}^{*} y_{t}\right) \beta-i\left(\dot{x}_{t}^{*} y_{t}^{*}-x_{t}^{*} \dot{y}_{t}^{*}\right) \beta^{\dagger}-\operatorname{Tr}_{\mathrm{BF}}[\beta, H] \mathcal{F} .
$$

The last three terms account for the explicit time dependence of the $\beta(t)$ related to the shift amplitudes $\mathcal{B}_{t}$ and to the Bogoliubov transformation (5). For the fermion operators $\alpha_{1}(t)$ and $\alpha_{-1}(t)$ the corresponding approximation reads

$$
\begin{aligned}
i \frac{d \alpha_{1}}{d t} & =\left[\alpha_{1}, H_{0}\right]+i\left(\dot{u}_{t}^{*} u_{t}+\dot{v}_{t}^{*} v_{t}\right) \alpha_{1}+i\left(\dot{u}_{t}^{*} v_{t}^{*}-u_{t}^{*} \dot{v}_{t}^{*}\right) \alpha_{-1} \\
i \frac{d \alpha_{-1}}{d t} & =\left[\alpha_{-1}, H_{0}\right]+i\left(\dot{u}_{t} u_{t}^{*}+\dot{v}_{t} v_{t}^{*}\right) \alpha_{-1}-i\left(\dot{u}_{t} v_{t}-u_{t} \dot{v}_{t}\right) \alpha_{1} .
\end{aligned}
$$

In these expressions the Hamiltonian written as $H_{0}(t)$ is taken as the effective mean-field Hamiltonian (cf. Ref. 99)

$$
\begin{aligned}
H_{0} & =\mathcal{P}^{\dagger}(t) H+\beta^{\dagger} \operatorname{Tr}_{\mathrm{BF}}[\beta, H] \mathcal{F}^{\prime}(t)-\beta T r_{\mathrm{BF}}\left[\beta^{\dagger}, H\right] \mathcal{F}^{\prime}(t) \\
& +\frac{\beta^{\dagger} \beta^{\dagger}}{2(1+2 \nu)} \operatorname{Tr}_{\mathrm{BF}}[\beta \beta, H] \mathcal{F}^{\prime}(t)-\frac{\beta \beta}{2(1+2 \nu)} \operatorname{Tr}_{\mathrm{BF}}\left[\beta^{\dagger} \beta^{\dagger}, H\right] \mathcal{F}^{\prime}(t) \\
& +\frac{\alpha_{1}^{\dagger} \alpha_{-1}}{\left(p_{-1}-p_{1}\right)} \operatorname{Tr}_{\mathrm{BF}}\left[\alpha_{-1}^{\dagger} \alpha_{1}, H\right] \mathcal{F}^{\prime}(t)+\frac{\alpha_{-1}^{\dagger} \alpha_{1}}{\left(p_{1}-p_{-1}\right)} \operatorname{Tr}_{\mathrm{BF}}\left[\alpha_{1}^{\dagger} \alpha_{-1}, H\right] \mathcal{F}^{\prime}(\Leftrightarrow \beta 7 .)
\end{aligned}
$$


The first term in this expression correspond to the lowest approximation according to ref. [9]. Note that $\mathcal{P}(t)$ is not an orthogonal projection, i.e. $\mathcal{P}^{\dagger}(t) \neq \mathcal{P}(t)$. The remaining terms, included here, represent correlation contributions to the effective mean-field.

Consistently with this approximation, the Green's function (34) is in lowest order just the unit operator

$$
\mathcal{G}\left(t, t^{\prime}\right)=\mathcal{I}
$$

so that the correlation density is approximated as

$$
\mathcal{F}^{\prime}(t)=\mathcal{G}^{(0)}(t, 0) \mathcal{F}^{\prime}(0)-i \int_{0}^{t} d t^{\prime} \mathcal{Q}\left(t^{\prime}\right) \mathcal{L F}_{0}\left(t^{\prime}\right) .
$$

For simplicity, we restrict ourselves now to initially uncorrelated states, i.e. $\mathcal{F}^{\prime}(0)=0$. The dynamical equations of the gaussian variables with correlation terms then become

$$
\begin{aligned}
i \frac{d}{d t}\left[\operatorname{Tr}_{\mathrm{BF}}\left(\mathcal{O}_{\mathrm{F}}(t) \mathcal{F}\right)\right]= & \begin{array}{l}
\operatorname{Tr}_{\mathrm{BF}}\left[\mathcal{O}_{\mathrm{F}}(t), H\right] \mathcal{F}_{0}(t)-i \operatorname{Tr}_{\mathrm{BF}}\left[\mathcal{O}_{\mathrm{F}}(t), H\right] \int_{0}^{t} d t^{\prime} \mathcal{Q}\left(t^{\prime}\right) \mathcal{L} \mathcal{F}_{0}\left(t^{\prime}\right) \\
\text { mean-field approx. }
\end{array} \\
i \frac{d}{d t}\left[\operatorname{Tr}_{\mathrm{BF}}\left(\mathcal{O}_{\mathrm{B}}(t) \mathcal{F}\right)\right]= & \begin{array}{l}
\operatorname{Tr}_{\mathrm{BF}}\left[\mathcal{O}_{\mathrm{B}}(t), H\right] \mathcal{F}_{0}(t)-i \operatorname{Tr}_{\mathrm{BF}}\left[\mathcal{O}_{\mathrm{B}}(t), H\right] \int_{0}^{t} d t^{\prime} \mathcal{Q}\left(t^{\prime}\right) \mathcal{L} \mathcal{F}_{0}\left(t^{\prime}\right) \\
\text { mean-field approx. } \quad \text { correlation term }
\end{array}
\end{aligned}
$$

where $\mathcal{O}_{\mathrm{F}}$ and $\mathcal{O}_{\mathrm{B}}$ correspond to fermion and boson gaussian variables, respectively.

The terms involving $\mathcal{F}_{0}(t)$ can be calculated from $H$ in quasi-particle basis and the projection operator $\mathcal{P}$ given by (27), (28) and (29). However, the traces in the last terms of (39) still can not be taken directly, since the quasiparticle operators in the integral and in the first commutator are at different times. Therefore, we use the approximation in which the time evolution of these operators is given by (35) and (36). Using (37), (20) and (23) we obtain

$$
i \frac{d \beta}{d t}=i\left(\dot{x}_{t}^{*} x_{t}-\dot{y}_{t}^{*} y_{t}\right) \beta+\omega\left(\left|x_{t}\right|^{2}+\left|y_{t}\right|^{2}\right) \beta \equiv F_{\beta}(t) \beta
$$




$$
\begin{aligned}
i \frac{d \alpha_{1}}{d t} & =\left[\frac{\epsilon}{2}\left(\left|u_{t}\right|^{2}-\left|v_{t}\right|^{2}\right)-\lambda\left(u_{t}^{*} v_{t} \mathcal{B}_{t}+u_{t} v_{t}^{*} \mathcal{B}_{t}^{*}\right)\right] \alpha_{1}+i\left(\dot{u}_{t}^{*} u_{t}+\dot{v}_{t}^{*} v_{t}\right) \alpha_{1} \equiv F_{\alpha_{1}}(t) \alpha_{1} \\
i \frac{d \alpha_{-1}}{d t} & =-\left[\frac{\epsilon}{2}\left(\left|u_{t}\right|^{2}-\left|v_{t}\right|^{2}\right)-\lambda\left(u_{t}^{*} v_{t} \mathcal{B}_{t}+u_{t} v_{t}^{*} \mathcal{B}_{t}^{*}\right)\right] \alpha_{-1}+i\left(\dot{u}_{t} u_{t}^{*}+\dot{v}_{t} v_{t}^{*}\right) \alpha_{-1} \equiv F_{\alpha_{-1}}(t) \alpha_{-1}
\end{aligned}
$$

From (40), (41) and (42) one can verify that the operators $\beta(t), \alpha(t)$ at different times are related as

$$
i \frac{d \beta}{d t} \equiv F_{\beta}(t) \beta \Longrightarrow \beta(t)=e^{i \varphi\left(t, t^{\prime}\right)} \beta\left(t^{\prime}\right)
$$

where the phase $\varphi\left(t, t^{\prime}\right)$ is given by

$$
\varphi\left(t, t^{\prime}\right)=-\int_{t^{\prime}}^{t} d \tau F_{\beta}(\tau)
$$

and

$$
\begin{gathered}
i \frac{d \alpha_{1}}{d t} \equiv F_{\alpha_{1}}(t) \alpha_{1} \quad \Longrightarrow \quad \alpha_{1}(t)=e^{i \xi_{\alpha}\left(t, t^{\prime}\right)} \alpha_{1}\left(t^{\prime}\right) \\
i \frac{d \alpha_{-1}}{d t} \equiv F_{\alpha_{-1}}(t) \alpha_{-1} \quad \Longrightarrow \alpha_{-1}(t)=e^{-i \xi_{\alpha}\left(t, t^{\prime}\right)} \alpha_{-1}(t)
\end{gathered}
$$

where the phase $\xi_{\alpha}\left(t, t^{\prime}\right)$ is

$$
\xi_{\alpha}\left(t, t^{\prime}\right)=-\int_{t^{\prime}}^{t} d \tau F_{\alpha}(\tau)
$$

Finally, the equations of motion that describe the present approximation to the collisional dynamics are

$$
\begin{aligned}
\dot{\nu} & =\lambda^{2}\left\{\left(u_{t}^{*} v_{t} y_{t}^{*}-u_{t} v_{t}^{*} x_{t}^{*}\right) I_{1}+\left(u_{t}^{* 2} y_{t}^{*}+v_{t}^{* 2} x_{t}^{*}\right) I_{2}+\left(u_{t}^{2} x_{t}^{*}+v_{t}^{2} y_{t}^{*}\right) I_{3}\right\}+ \\
& + \text { C.C. }
\end{aligned}
$$




$$
\begin{aligned}
\frac{d}{d t}\left(p_{1}-p_{-1}\right) & =2 \lambda^{2}\left\{-\left(u_{t}^{* 2} x_{t}+v_{t}^{* 2} y_{t}\right) I_{3}^{*}+\left(u_{t}^{* 2} y_{t}^{*}+v_{t}^{* 2} x_{t}^{*}\right) I_{2}\right\} \\
& + \text { C.C. } \\
i\left(p_{1}-p_{-1}\right)\left(\dot{u}_{t} v_{t}-u_{t} \dot{v}_{t}\right) & =\left(p_{1}-p_{-1}\right)\left[\epsilon u_{t} v_{t}+\lambda\left(u_{t}^{2} \mathcal{B}_{t}^{*}-v_{t}^{2} \mathcal{B}_{t}\right)\right]+ \\
& -2 i \lambda^{2}\left(u_{t}^{*} v_{t} x_{t}-u_{t} v_{t}^{*} y_{t}\right) I_{3}^{*}-2 i \lambda^{2}\left(u_{t} v_{t}^{*} x_{t}^{*}-u_{t}^{*} v_{t} y_{t}^{*}\right) I_{2}+ \\
& +i \lambda^{2}\left(u_{t}^{2} y_{t}+v_{t}^{2} x_{t}\right) I_{1}^{*}+i \lambda^{2}\left(u_{t}^{2} x_{t}^{*}+v_{t}^{2} y_{t}^{*}\right) I_{1} \\
i(1+2 \nu)\left(\dot{x}_{t}^{*} y_{t}^{*}-x_{t}^{*} \dot{y}_{t}^{*}\right) & =-(1+2 \nu) 2 \omega x_{t}^{*} y_{t}^{*}-2 i \lambda^{2}\left(u_{t}^{*} v_{t} y_{t}^{*}-u_{t} v_{t}^{*} x_{t}^{*}\right) I_{1}^{*}+ \\
& +2 i \lambda^{2}\left(u_{t}^{* 2} y_{t}^{*}+v_{t}^{* 2} x_{t}^{*}\right) I_{3}^{*}+2 i \lambda^{2}\left(u_{t}^{2} x_{t}^{*}+v_{t}^{2} y_{t}^{*}\right) I_{2}^{*}
\end{aligned}
$$

where

$$
\begin{aligned}
& I_{1}=\int_{0}^{t} d t^{\prime}\left(u_{t^{\prime}} v_{t^{\prime}}^{*} y_{t^{\prime}}-u_{t^{\prime}}^{*} v_{t^{\prime}} x_{t^{\prime}}\right)\left[p_{1}\left(1-p_{1}\right)+p_{-1}\left(1-p_{-1}\right)\right]_{t^{\prime}} e^{i \varphi\left(t, t^{\prime}\right)} \\
& \left.I_{2}=\int_{0}^{t} d t^{\prime}\left(u_{t^{\prime}}^{2} y_{t^{\prime}}+v_{t^{\prime}}^{2} x_{t^{\prime}}\right)\left[p_{-1}\left(1-p_{1}\right)+\left(p_{-1}-p_{1}\right) \nu\right]_{t^{\prime}} e^{-i\left(2 \xi\left(t, t^{\prime}\right)+\varphi\left(t, t^{\prime}\right)\right.}\right)(51) \\
& I_{3}=\int_{0}^{t} d t^{\prime}\left(u_{t^{\prime}}^{* 2} x_{t^{\prime}}+v_{t^{\prime}}^{* 2} y_{t^{\prime}}\right)\left[p_{1}\left(1-p_{-1}\right)+\left(p_{1}-p_{-1}\right) \nu\right]_{t^{\prime}} e^{i\left(2 \xi\left(t, t^{\prime}\right)-\varphi\left(t, t^{\prime}\right)\right.}(52)
\end{aligned}
$$

Using the phase equation (43) one can write the integral $I_{1}$ as

$$
I_{1}=e^{-I_{\beta}} I_{e}^{(1)}(t)
$$

where

$$
I_{\beta}(t)=\int_{0}^{t} d t^{\prime} F_{\beta}\left(t^{\prime}\right)
$$




$$
\begin{aligned}
I_{e}^{(1)}(t) & =\int_{0}^{t} d t^{\prime}\left(u_{t^{\prime}} v_{t^{\prime}}^{*} y_{t^{\prime}}-u_{t^{\prime}}^{*} v_{t^{\prime}} x_{t^{\prime}}\right)\left[p_{1}\left(1-p_{1}\right)+p_{-1}\left(1-p_{-1}\right)\right]_{t^{\prime}} e^{i \varphi\left(t^{\prime}, \varphi\right.} \\
F_{\beta} & =i\left(\dot{x}_{t}^{*} x_{t}-\dot{y}_{t}^{*} y_{t}\right)+\omega\left(\left|x_{t}\right|^{2}+\left|y_{t}\right|^{2}\right)
\end{aligned}
$$

To solve the equation (53) we write differential equation for the integrals $I_{\beta}$ and $I_{e}^{(1)}$

$$
\begin{aligned}
\dot{I}_{\beta} & =i\left(\dot{x}_{t}^{*} x_{t}-\dot{y}_{t}^{*} y_{t}\right)+\omega\left(\left|x_{t}\right|^{2}+\left|y_{t}\right|^{2}\right) \\
\dot{I}_{e}^{(1)} & =\left(u_{t} v_{t}^{*} y_{t}-u_{t}^{*} v_{t} x_{t}\right)\left[p_{1}\left(1-p_{1}\right)+p_{-1}\left(1-p_{-1}\right)\right]_{t} e^{-I_{\beta}}
\end{aligned}
$$

Using the other phase equation we obtain, from the integrals $I_{2}$ and $I_{3}$, the following differential equations

$$
\begin{aligned}
\dot{I}_{e}^{(2)} & =\left(u_{t}^{2} y_{t}+v_{t}^{2} x_{t}\right)\left[p_{-1}\left(1-p_{1}\right)+\left(p_{-1}-p_{1}\right) \nu\right]_{t} e^{-i\left(2 I_{\alpha}+I_{\beta}\right)} \\
\dot{I}_{e}^{(3)} & =\left(u_{t}^{* 2} x_{t}+v_{t}^{* 2} y_{t}\right)\left[p_{1}\left(1-p_{-1}\right)+\left(p_{1}-p_{-1}\right) \nu\right]_{t} e^{i\left(2 I_{\alpha}-I_{\beta}\right)} \\
\dot{I}_{\alpha} & =i\left(\dot{u}_{t}^{*} u_{t}+\dot{v}_{t}^{*} v_{t}\right)+\frac{\epsilon}{2}\left(\left|u_{t}\right|^{2}-\left|v_{t}\right|^{2}\right)-\lambda\left(u_{t}^{*} v_{t} \mathcal{B}_{t}+u_{t} v_{t}^{*} \mathcal{B}_{t}^{*}\right)
\end{aligned}
$$

Thus, we can integrate the equations (57-58) and (59-61) together with the equations (46-49). These equations describe the time evolution of the gaussian variables in the collisional approximation.

\section{Results and Discussion}

We give below results obtained for two relevant observables associated with the spin subsystem of the Jaynes-Cummings Hamiltonian: the atomic inversion, $\left\langle\sigma_{3}\right\rangle_{t}$, and the "intrinsic" atomic inversion, defined in terms of the 
projection of $\vec{\sigma}$ along a unit vector pointing along the direction of the spin vector, $\langle\vec{\sigma}\rangle /|\langle\vec{\sigma}\rangle|$, which will be denoted by $\left\langle\sigma_{p}\right\rangle_{t}[12]$. The latter quantity corresponds in fact to the degree of polarization of the spin $1 / 2$ subsystem, and hence is directly related to the purity of the corresponding reduced density. Thus any time dependence of this quantity implies non-isoentropic behavior of the subsystem dynamics. In terms of the Bogoliubov parameters, the mean values of these observables are given by

$$
\begin{gathered}
\left\langle\sigma_{3}\right\rangle_{t}=\left(p_{1}-p_{-1}\right) \cos 2 \theta \\
\left\langle\sigma_{p}\right\rangle_{t}=\left(p_{1}-p_{-1}\right)
\end{gathered}
$$

where we use the parametrization

$$
\begin{aligned}
& u_{t}=\cos \theta \\
& v_{t}=e^{-i \delta} \sin \theta .
\end{aligned}
$$

A typical example of the results obtained within the present approximations for the time evolution of these objects is shown in the figures below. They have been obtained by solving numerically the dynamical equations 46 49) and are compared with the corresponding exact solutions of the model. Figure 1 shows the time evolution of the atomic inversion $\left\langle\sigma_{3}\right\rangle_{t}$ when the atom is initially prepared in the excited state $\left(p_{1}(0)=1, p_{-1}(0)=0\right)$ and the field is initially in the coherent state constructed as the vacuum of the displaced annihilation operator $b\left(x_{t=0}=1, y_{t=0}=0\right.$ and $\left.\nu(0)=0\right)$. The mean photon number $\mathcal{B}_{t=0}^{2}$ is 25 in this example. We use units such that $\epsilon=\omega=1$ and $\lambda=0.5$. The evolution of the intrinsic polarization for the same initial conditions is shown in Figure 2.

The mean-field approximation amounts to ignoring the integrals $I_{1}, I_{2}$ and $I_{3}$, which are set to zero at all times in this case. This causes the occupation probabilities $\nu, p_{1}$ and $p_{-1}$ to become time-independent, so that there are no correlations developing between the subsystems. As a consequence, there is no depolarization and the inversion undergoes Rabi oscillations with constant amplitude. In the collisional approximation a modulation of the amplitude of the Rabi oscillations is obtained from the depolarization resulting from the approximate collisional corrections to the mean field approximation, included 
in Eqs. (46) to (52), which allow for intersubsystem correlations to develop. The effectiveness of the present treatment of these corrections can be judged by comparing its results with the exact solution. As can be seen in the figures, the approximate solution is quantitatively adequate up to times comparable with the depolarization time.

We may infer from this that the inclusion of the present approximation of the collision integrals in the dynamical equations is not only fundamental to generate the qualitative behavior associated with the decorrelation process related to the initial damping of the Rabi oscillations, but also successfully describes such effects quantitatively over a time span covering at least several Rabbi periods. Improvements of the collisional approximation implemented here are however needed if one wishes to extend time range of quantitative reliability.

Finally, we briefly comment on the extension of the present treatment to $3+1$ dimensions in a field-theoretical context (the scalar plasma [5]). In general, an entirely analogous procedure will apply also to this case, if only one expands the field operators in terms of the eigenfunctions of the corresponding extended one-fermion and one-boson densities. This is in general a time-dependent basis whose evolution will be determined by generalized forms of Eqs. (48) and (49). The case of a spatially uniform system is particularly simple, since in this case the extended densities are diagonal in a plane-wave representation. It can be handled therefore in complete analogy with the $0+1$ dimensional case if one uses a momentum expansion of the field operators [11]. As expected, the usual infinities of quantum field theory show up and can be absorbed by introducing appropriate counterterms. Work in this line is under way.

\section{Acknowledgements}

This work was supported by Conselho Nacional de Desenvolvimento Científico e Tecnológico (CNPq), Brazil. 


\section{References}

[1] M.C. Nemes and A.F.R. de Toledo Piza, Physica 137A (1986) 367.

[2] R. Jackiw, Physica A158 (1989) 269

O. Éboli, S.Y. Pi and R. Jackiw, Phys. Rev D37 (1988) 3557.

[3] R. Omnès, Rev. Mod. Phys. 64 (1992) 339

H. Moya-Cessa, V. Buz̃ek, M.S. Kim and P.L. Knight, Phys. Rev. A48 (1993) 3900.

[4] E.T. Jaynes and F.W. Cummings, Proc. IEEE 51 (1963) 89.

[5] G. Kalman, Phys. Rev. 161 (1967) 156

G. Kalman, Phys. Rev. D9 (1984) 2690.

[6] P. Ring and P. Schuck, The Nuclear Many-body Problem, Springer-Verlag.

[7] L.C. Yong and A.F.R. de Toledo Piza, Mod. Phys. Lett. A5 (1990) 1605.

[8] C.R. Willis and R.H. Picard, Phys. Rev. A9 (1974) 1343.

[9] P. Buck, H. Feldmeier e M.C. Nemes, Ann. of Phys. 185 (1988) 170.

[10] J. Des Cloiseaux, in Many Body Physics, C. de Witt and R. Balian, eds., Gordon and Breach, N.Y., 1968 pp.5-36.

[11] L.C. Yong, Doctoral Thesis, University of São Paulo, 1991 (unpublished) L.C. Yong and A.F.R. de Toledo Piza, Phys. Rev. D 46 (1992) 742.

[12] M. L. C. Rabello and A. F. R. de Toledo Piza, Rev. Bras. Fis. 14 (1984) 353. 


\section{Figure Captions}

Fig.1. Time evolution of the atomic inversion with $\lambda=0.5$. Initial conditions: $\nu=0, x_{t}=1, y_{t}=0,\left|\mathcal{B}_{t}\right|=5$ (coherent state); $p_{1}=1, p_{-1}=0$. Full line: exact solution; dot-dashed line: collisional approximation; dashed line: mean-field approximation.

Fig.2. Time evolution of the intrinsic polarization. The initial conditions are the same as Fig. 1. Full line: exact solution; dot-dashed line: collisional approximation. 
Figure 1

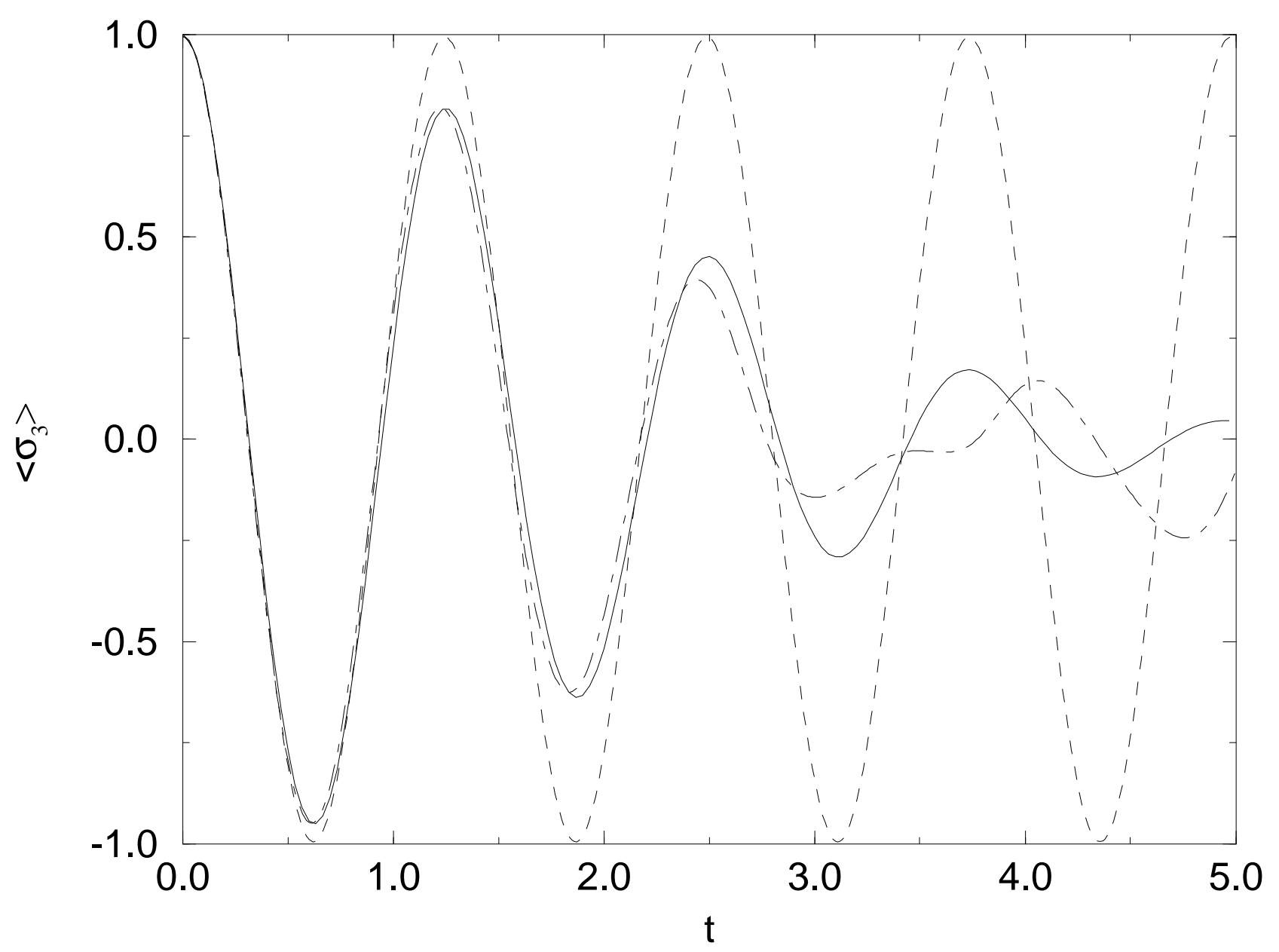


Figure 2

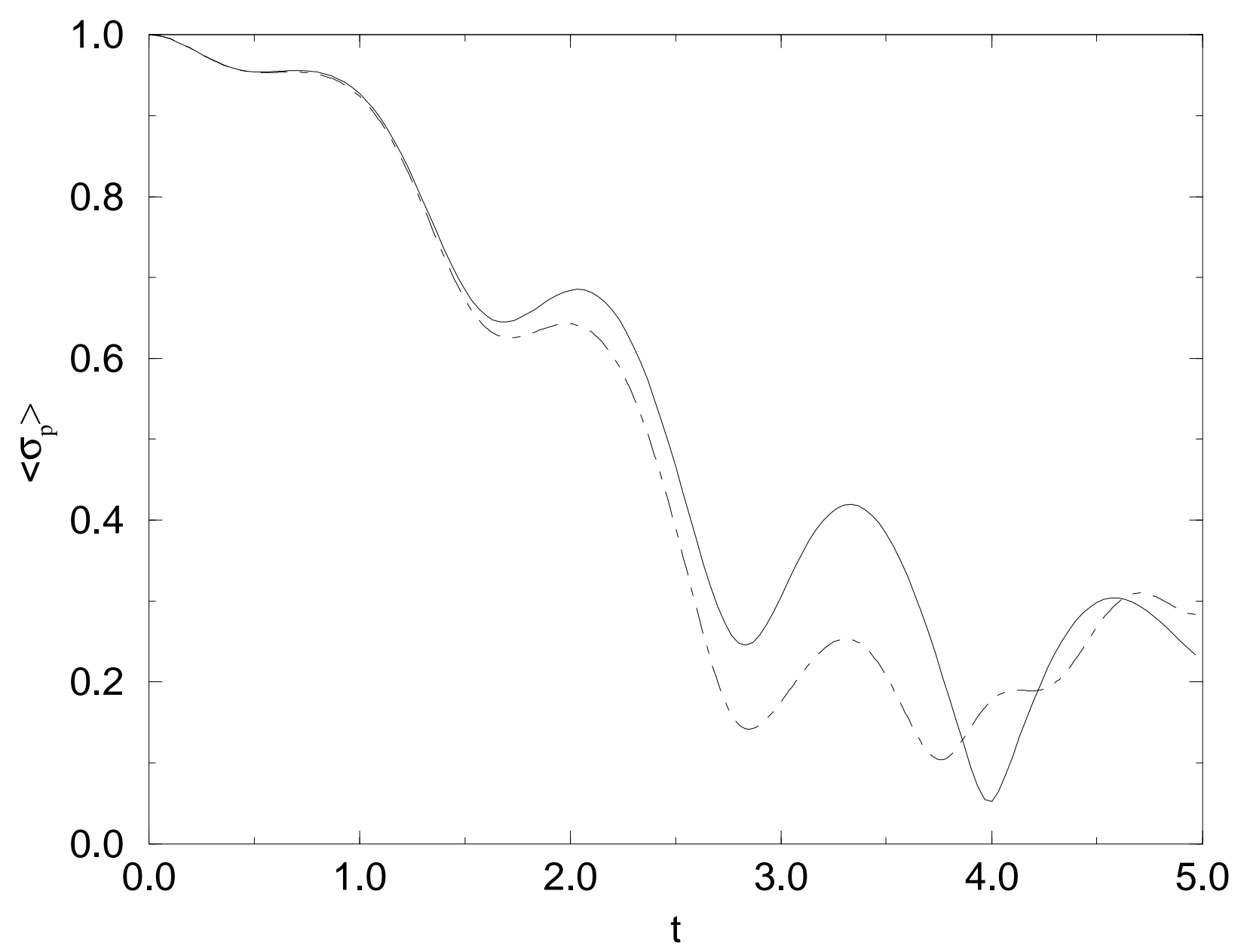

